\title{
Miniaturization of a-Si guided mode resonance filter arrays for near-IR multi- spectral filtering
}

Cite as: Appl. Phys. Lett. 117, 111106 (2020); https://doi.org/10.1063/5.0024302

Submitted: 06 August 2020 . Accepted: 03 September 2020 . Published Online: 18 September 2020

Ryan C. Ng (D), Juan C. Garcia, Julia R. Greer, and Katherine T. Fountaine
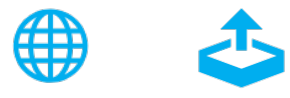

\section{ARTICLES YOU MAY BE INTERESTED IN}

Epsilon near-zero all-optical terahertz modulator

Applied Physics Letters 117, 111101 (2020); https://doi.org/10.1063/5.0012206

Milliwatt power $233 \mathrm{~nm}$ AlGaN-based deep UV-LEDs on sapphire substrates

Applied Physics Letters 117, 111102 (2020); https://doi.org/10.1063/5.0015263

Transparent phototransistor with high responsivity, sensitivity, and detectivity from heterojunction metal oxide semiconductors

Applied Physics Letters 117, 111103 (2020); https://doi.org/10.1063/5.0014562

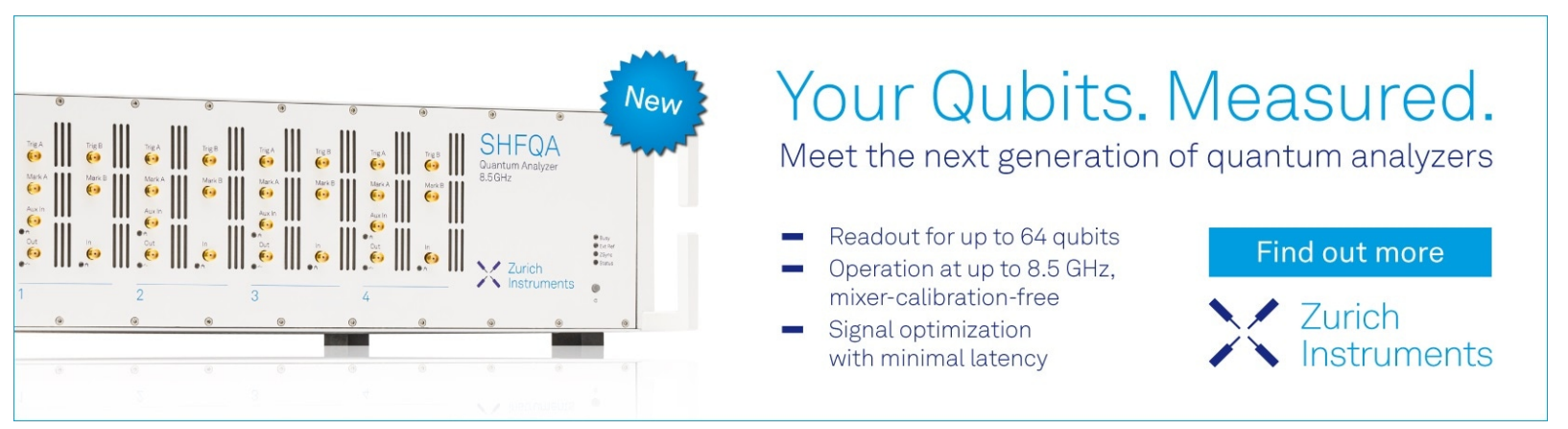




\title{
Miniaturization of a-Si guided mode resonance filter arrays for near-IR multi-spectral filtering
}

\author{
Cite as: Appl. Phys. Lett. 117, 111106 (2020); doi: 10.1063/5.0024302 \\ Submitted: 6 August 2020 - Accepted: 3 September 2020 . \\ Published Online: 18 September 2020
}

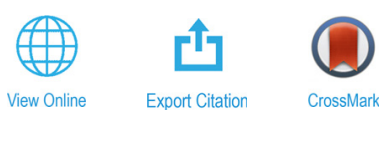

Ryan C. Ng, ${ }^{1, a)}$ (D) Juan C. Garcia, ${ }^{2}$ Julia R. Greer, ${ }^{3}$ and Katherine T. Fountaine ${ }^{2}$

\begin{abstract}
AFFILIATIONS
'Division of Chemistry and Chemical Engineering, California Institute of Technology, Pasadena, California 91125, USA

${ }^{2}$ NG Next, Northrop Grumman Corporation, One Space Park, Redondo Beach, California 90278, USA

${ }^{3}$ Division of Engineering and Applied Sciences, California Institute of Technology, Pasadena, California 91125, USA
\end{abstract}

a) Author to whom correspondence should be addressed: reng@caltech.edu

\begin{abstract}
Sub-wavelength periodic arrays exhibit narrow near-unity reflection bands that arise from guided mode resonances. These resonances have extremely high quality factor (i.e., narrow band features) and are ideal for filtering applications. A high quality factor requires many periods, causing large lateral footprints that limit an imaging system's spatial resolution. We present a 1D ultra-thin $(<100 \mathrm{~nm})$ compact finite design of seven periods of amorphous Si slabs with subwavelength periodicity surrounded by Al mirrors, which allow the finite array to approximate an infinite array and enabling a small footprint $(\sim 5 \mu \mathrm{m})$, for near-infrared applications $(\lambda=800-2000 \mathrm{~nm})$. We demonstrate spectral tunability (amplitude, bandwidth, and peak location) via geometric parameter variation and demonstrate the performance of these filters both in experiment and in simulation. This work miniaturizes guided-mode resonance filters, previously limited by extremely large footprints, while being relatively cheap and simple to fabricate compared to many existing designs.
\end{abstract}

Published under license by AIP Publishing. https://doi.org/10.1063/5.0024302

Spectral imaging is a powerful imaging technique that allows for remote noncontact sensing, as every chemical species emits a unique spectral signature that can be used to differentiate them from one another. This tool has a wide range of applications, including environmental tracking, ${ }^{1-5}$ food inspection, ${ }^{6,7}$ biology and medicine, ${ }^{8,9}$ surveillance, ${ }^{10}$ among others. ${ }^{11,12}$ Realization of these applications requires a suite of filters that exhibit spectral bands at varying wavelengths, with filter characteristics such as narrow bandwidth or high spectral resolving power (small $\Delta \lambda / \lambda$ ) and high signal-to-noise ratio or in-band transmission/reflection amplitude to maximize the spectral resolution, and small footprint to maximize the spatial resolution. Traditionally, the standards for bandpass filters are absorption filters and dichroic filters. Absorption filters suffer from ultraviolet or high-temperature degradation in the long term and have relatively broad bandwidths. Interference filters provide greater control over the spectral characteristics, but have a large footprint, necessitating scanning imaging methods, and have total thicknesses $>1 \mu \mathrm{m}$ with 10 's to 100 's of layers, requiring a complex fabrication process. ${ }^{13}$ Thick filters also result in a greater distance for optical crosstalk to occur and a decrease in imaging device performance because as the lateral size of a pixel (and the microlens) decreases, the total filter/circuitry stack in a pixel must also decrease to ensure that the microlens focal length is smaller than that of the imaging lens. ${ }^{14,15}$

Many other filter designs have been reported in the literature, including designs based on plasmonic arrays, ${ }^{16-22}$ Fabry-Pérot resonators, ${ }^{23-25}$ and guided mode resonance (GMR) filters. ${ }^{26-32}$ Plasmonic arrays operate via the interference of surface plasmon polaritons (SPPs) between adjacent holes/slits. These often suffer from low efficiency due to metal absorption. Fabry-Pérot resonators form a cavity of low-absorbing material between two reflective films with a passband on resonance. In these designs, while small lateral footprints are possible, bandwidths of $>100 \mathrm{~nm}$ are typically observed, sufficient for RGB imaging, but not for multi- or hyper-spectral imaging. In GMR filters, long-range periodicity enables an incident wave to couple into a laterally propagating waveguide mode within the array via a grating vector, and subsequently reradiate, which manifests as a narrowband, nearunity rapid spectral variation. ${ }^{33-36}$ In addition to the GMR, other formalisms have been proposed to treat these rapid spectral variations such as the interference of axially propagating waveguide modes ${ }^{37-39}$ and the interference of periodic scatterers. ${ }^{40}$ Sturmberg et al. attempted to reconcile these different formalisms by describing these spectral features as an asymmetric Fano resonance that results from 
the interference of an axially propagating Fabry-Pérot mode and inplane propagating grating vector-coupled waveguide mode. We previously compared these formalisms ${ }^{41}$ and concluded that the guided mode resonance formalism is the most generally applicable formalism and, thus, is the formalism we incorporate in our work. Many aforementioned applications require narrowband filters in the infrared (IR), for which the characteristics of plasmonic arrays and Fabry-Pérot resonators are insufficient. However, GMR filters, which have been demonstrated in the IR, ${ }^{34,41-51}$ possess spectral characteristics ideal for spectral filtering. While GMR-based filters possess high resolving power, they require a significant number of periods, leading to pixel sizes on the order of 10's to 100's of micrometers in the IR, much too large to provide high spatial resolution in compact imaging systems. ${ }^{52-56}$ This relationship between spectral resolving power and lateral periodicity is a typical trade-off for periodic filter designs. ${ }^{5}$

In GMR-based designs, many periods are required as once an incident wave is scattered into a laterally propagating waveguide mode, it must propagate for a sufficient number of periods until it scatters out, manifesting as the GMR phenomenon. In a finite lattice, if the laterally propagating mode reaches the array edge prior to scattering out, it can out-couple and the energy is lost. Thus, high quality factor modes often are not observable in finite lattices. ${ }^{56}$ These edge losses can be suppressed by emulating infinite periodicity via reflective boundaries outside the array such that the array essentially unfolds into an infinitely periodic structure. This strategy has been experimentally demonstrated in the visible through near-IR to be effective for small aperture size GMR designs using distributed Bragg reflectors (DBRs) as the reflective boundaries. ${ }^{56,58,59}$ However, the many layers of a DBR greatly increase the lateral footprint and are unideal in a spectral imaging system. In the radio wave regime, a single reflective mirror was used at each boundary in place of DBRs in an all-dielectric GMR design. ${ }^{60}$ In this work, we employ this strategy in the near-IR regime.

We report the optical properties of a 1D seven-period compact amorphous $\mathrm{Si}(\mathrm{a}-\mathrm{Si})(n \approx 3.5)$ grating surrounded on two sides with reflective $\mathrm{Al}$ mirrors, embedded in $\mathrm{SiO}_{2}(n \approx 1.4)$, designed for multispectral imaging applications in the near-infrared. While we restrict this work to a $1 \mathrm{D}$ design, the ideas here can be easily extended to $2 \mathrm{D}$ for polarization-independence (see Fig. S1 in the supplementary material). This design also has a lower loss than plasmonic-based designs, is ultra-thin, lightweight, UV-resistant, thermally stable, stable against humidity, CMOS compatible, has low material usage, and uses redundant processing for different filters. GMR spectral characteristics are dependent on the incidence angle due to conservation of momentum for in-coupling, with a decrease in peak amplitude and peak shifting as the incidence angle is increased; thus, we restrict this design to normal incidence. ${ }^{41,45}$ Figure 1 (a) presents a schematic of the proposed design. Here, the incident light is transverse electric (TE) (electric field parallel to grating lines). As the incident light wave is scattered via the periodicity of the seven-period grating into a laterally propagating leaky waveguide mode, the $\mathrm{Al}$ mirrors allow this laterally propagating mode to reflect back and continue propagating until it scatters back out, giving rise to the GMR. These filters are subwavelength; only the zeroth diffraction order can propagate, allowing only specular reflection/transmission. The grating slab height, periodicity, fill fraction of high index material (a-Si), and wall height are denoted by $h, a, f$, and $d$, respectively. A spacer region, $s$, defined as the distance
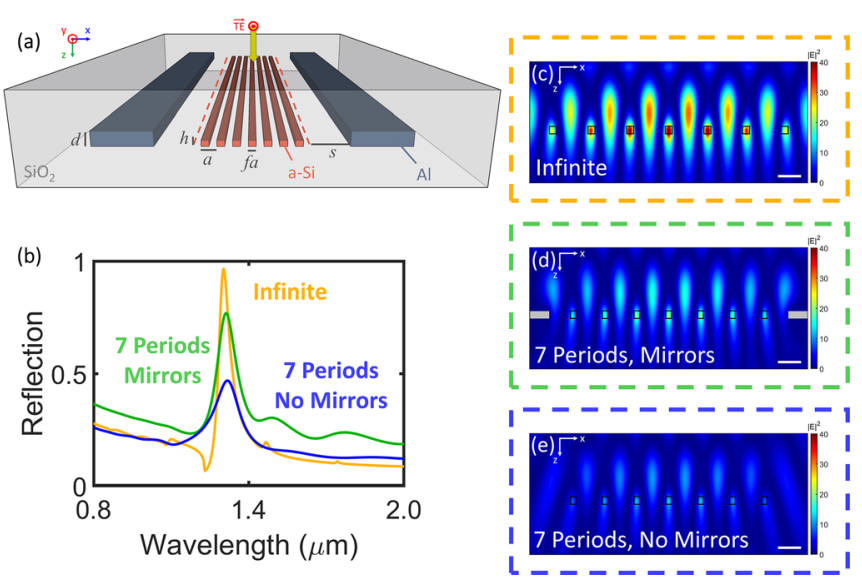

FIG. 1. Design of a seven-period finite filter. (a) Schematic of the 1D compact a-Si GMR filter with 7 periods of a-Si slabs surrounded by Al mirrors embedded in $\mathrm{SiO}_{2}$. The array of a-Si slabs has thickness, $h$, periodicity, a, fill fraction, $f$, and wall height, d. A spacer region, $s$, denotes additional space between the filter and mirror. The incident light is TE polarization (red text), where the electric field is parallel to the grating lines. Schematic is not to scale. (b) Simulated reflectivity spectra comparing the GMR filter in three different design variations: an infinite array (no mirrors), the finite 7-period a-Si slab design with Al mirrors illustrated in (a), and a finite 7-period a-Si slab design without Al mirrors. Their respective electric field intensity profiles of a filter cross section are given in (c)-(e). Black boxes represent the a-Si slabs, while gray squares represent the Al mirrors. Scale bars are $500 \mathrm{~nm}$.

half a period from the center of the outermost grating slab to the inner mirror edge, is incorporated to ensure that the reflected wave is in phase. To determine the optimal value for these parameters, we utilized nonlinear optimization by mesh adaptive direct search (NOMAD), a method ideal for metamaterial optimization that iteratively alternates between global and local searches to efficiently find the global optimum. ${ }^{57,61-63}$ From NOMAD, an optimized 7-period filter was found with the parameters $h=64 \mathrm{~nm}, a=831 \mathrm{~nm}, f=0.18, d=61 \mathrm{~nm}$, and $s=215 \mathrm{~nm}$, and used as the nominal design for this study. With a lateral footprint of $6.2 \mu \mathrm{m}$ and a thickness of only $64 \mathrm{~nm}$, these filters are compact and ultra-thin, which is advantageous in an imaging device. $^{14,15}$

Figure 1(b) compares the spectra of three finite filter designs generated by finite-difference time domain (FDTD) simulations that motivate the inclusion of mirrors. The optimized compact 7-period design with $\mathrm{Al}$ mirrors (green) depicted in Fig. 1(a) exhibits lower inband reflection and greater full-width at half maximum (FWHM) than the infinite case (yellow), but greater in-band reflection and slightly lower FWHM than a compact 7-period design without mirrors (blue). For the proposed finite filter design (green), the resonance occurs at $1313 \mathrm{~nm}$ in $\mathrm{SiO}_{2}$ (effective wavelength of $\sim 912 \mathrm{~nm}$ ), making the structure barely sub-wavelength. While this design exhibits resonances in reflection, we envision their use as subtractive filters rather than requiring extraction of the reflected signal. As GMR reflection peaks often possess a Fano line shape, or asymmetric background, we define FWHM as the bandwidth at half of the maximum amplitude, rather than half of the amplitude minus the background, leading to an underestimation of performance. To match the peak amplitude of our proposed design or the infinite design using a mirrorless finite design, approximately 17 periods and 141 periods are required, respectively, 
for this set of geometric parameters as the peak increases in amplitude and narrows with an increasing number of periods (see Fig. S2 in the supplementary material). At the sacrifice of $19.9 \%$ reflection and an FWHM increase in $55.2 \mathrm{~nm}$, the lateral footprint of this finite 7-period filter has decreased by more than a factor of 18 when compared to the 141 periods required to match the performance of an infinite array and by more than a factor of 2 when compared to the 17 periods required to obtain a similar amplitude response in a finite design without mirrors. Filter designs utilizing reflective mirror boundaries incorporating as few as 3 periods still exhibit a reflection peak (see Fig. S3 in the supplementary material). In this case, although peak amplitude decreases, the peak broadens, and the background reflectivity increases, this performance is still significant as extremely small filter footprints $(<3 \mu \mathrm{m})$ can be obtained if the lower signal-to-noise ratio is acceptable for a given application. For each of the scenarios in Fig. 1 (b), the associated electric field intensity profiles are presented in Figs. 1(c)-1(e). In Fig. 1(c), a clear enhancement of the electric field is observed for the infinite case, indicative of the GMR phenomenon. Expressing the order-mode resonance as $\mathrm{TE}_{\mathrm{m}, \mathrm{v}}$, where $m$ is the generating diffraction order and $v$ is the resultant mode, this GMR's ordermode resonance is $\mathrm{TE}_{1,0}{ }^{45}$ The finite case with mirrors [Fig. 1(d)] shows greater field enhancement and less loss relative to the mirrorless finite case [Fig. 1(e)], though this enhancement is not as strong as the infinite case. These results clearly demonstrate the advantage of incorporating mirrors in a finite GMR filter design.

As we previously demonstrated experimentally and computationally for an infinite array of a-Si nanopillars embedded in $\mathrm{SiO}_{2}$, GMR spectral characteristics such as spectral location, amplitude, and bandwidth (FWHM) can be controlled via the variation of array geometric parameters. ${ }^{41}$ We demonstrate GMR sensitivity in the current designs with a similar analysis. Figure 2 presents intensity plots from
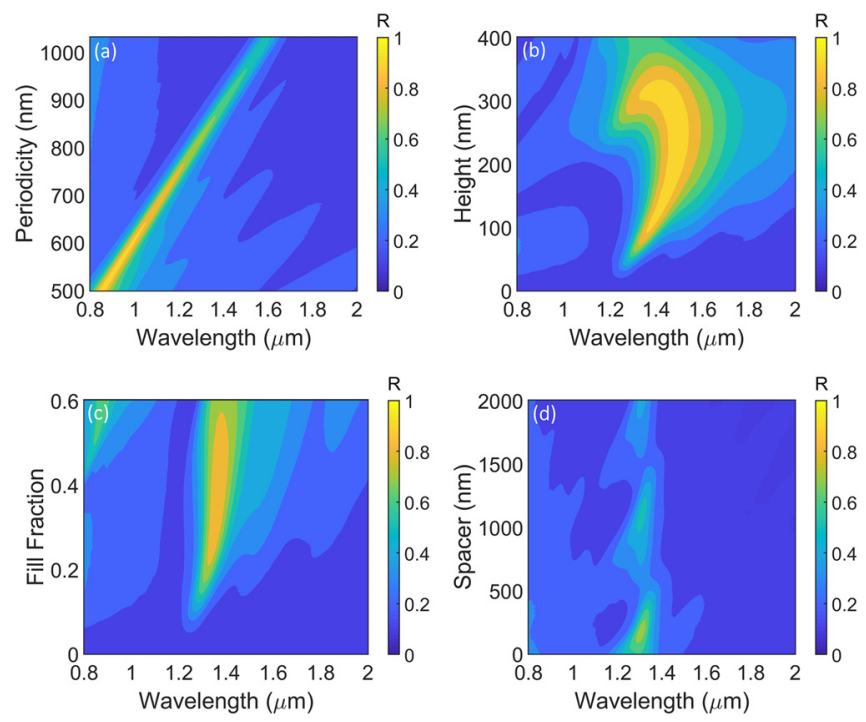

FIG. 2. Intensity plots of the simulated reflectivity (color axis) vs wavelength (x-axis) and structure dimensions (y-axis); (a) $h=64 \mathrm{~nm}, f=0.18, s=215 \mathrm{~nm}$, variable $a$; (b) $a=831 \mathrm{~nm}, f=0.18, s=215 \mathrm{~nm}$, variable $h$; (c) $a=831 \mathrm{~nm}$, $h=64 \mathrm{~nm}, s=215 \mathrm{~nm}$, variable $f$; (d) $a=831 \mathrm{~nm}, h=64 \mathrm{~nm}, f=0.18$, variable $s$.
FDTD simulations in the near-IR, where geometric parameters $a, h, f$, and $s$, were varied one at a time while the others were held constant. The strongest parameter to control resonance spectral location is periodicity [Fig. 2(a)], with a GMR peak that shifts $\sim 800 \mathrm{~nm}$ when varying $a$ between 500 and $1000 \mathrm{~nm}$. This tunability is important as a spectral imaging system requires a suite of filters that each filter a separate wavelength. While reflection amplitude decreases with increasing periodicity, this effect can be counteracted via an increase in the height [Fig. 2(b)] or fill fraction [Fig. 2(c)] of the a-Si slabs, though significant parameter variation can cause adjacent modes to overlap, as observed in Fig. 2(b), with a significant broadening and blue-shifted tail at greater $h$, as the resonance interacts with a mode at lower wavelengths. Between the variation of height or the variation of fill fraction, only fill fraction variation is realistic for the parallel fabrication of all filters on a single chip in a complete spectral imaging system. All filters on a single chip have the same height fixed by the thickness of the material deposited. For each variation, there is an optimal range of parameters, outside of which the resonance disappears, though this range can be tuned by simultaneously varying other parameters. Figure 2(d) shows the effect of the spacer, $s$, to ensure that the laterally propagating mode within the array does not destructively interfere with itself. An optimal range exists for $s$, where the resonance amplitude is the highest, outside of which the amplitude quickly decreases. When $s$ is increased by a factor of the period, we observe a return of the resonance, with reduced amplitude each time due to loss as the laterally propagating mode propagates further to reach the mirrors. These results demonstrate the ability to passively tune spectral characteristics of the finite 7-period GMR filter via geometric parameter variation.

With this design methodology, we fabricated five a-Si gratings with varying periodicity and measured their transmission to verify simulations (Fig. 3). Figures 3(a) and 3(b) display the experimental and simulated transmission spectra, respectively, for the fabricated arrays at normal incidence (see Methods in the supplementary material). The spectral characteristics of each array are summarized in Table S1 (see Table S1 in the supplementary material). Experiment and simulation follow similar qualitative trends, with red-shifting, peak broadening, and increase in peak transmission with increasing periodicity, and good alignment of bandwidths and peak spectral location, though the peaks are weaker in experiment. We observe FWHM values from 140.5 to $205.1 \mathrm{~nm}$ in experiment and from 102.7 to
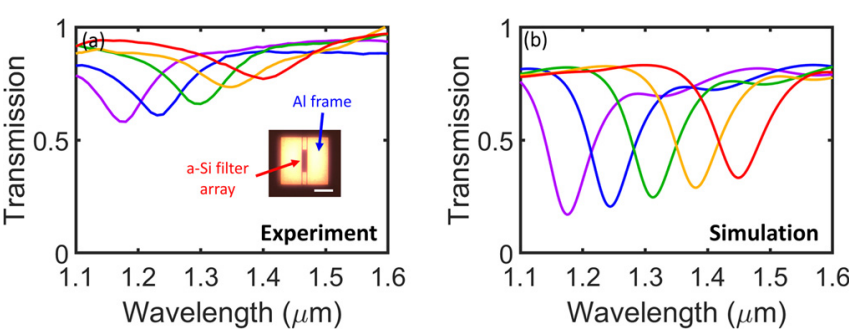

FIG. 3. Comparison of (a) experimental and (b) simulated transmission measurements for variable periodicity. The arrays have NOMAD optimized geometric parameters of $h=64 \mathrm{~nm}, f=0.18, d=61 \mathrm{~nm}, s=215 \mathrm{~nm}$, and variable $a=731 \mathrm{~nm}$ (purple), $781 \mathrm{~nm}$ (blue), $831 \mathrm{~nm}$ (green), $881 \mathrm{~nm}$ (yellow), and $931 \mathrm{~nm}$ (red). The inset in (a) is a $100 \mathrm{X}$ magnification microscope image of a filter (dark region) surrounded by a $50 \times 50 \mu \mathrm{m}$ reflective $\mathrm{Al}$ frame (bright region). The inset scale bar is $20 \mu \mathrm{m}$. 
$120.6 \mathrm{~nm}$ in simulation (overestimation of the actual FWHM and underestimation of their performance due to defining the FWHM as half of the peak reflection, defined previously). The transmission dips are as low as $58.1 \%$ experimentally, compared to $17.4 \%$ in simulation. We attribute this efficiency loss to fabrication imperfections, experimental normalization, and alignment errors in fabrication and measurement (angle and polarization). Figure 2(d) demonstrates that a misalignment of the spacer region quenches the expected resonance, even with a misalignment of only 10's of nm's. We note that a tradeoff exists between peak intensity and FWHM within the arrays, as determined by the figure of merit weighting within the NOMAD optimization scheme. The intensity and FWHM values we observe are a result of the specific weighting used here, but the modification of this figure of merit allows for optimization of a particular spectral characteristic. However, these results experimentally demonstrate GMRs with acceptable SNR for spectral imaging with this design.

With a $\sim 5 \mu$ m lateral footprint, this design has a pixel size on par with commercial CMOS arrays. As filter size decreases, optical crosstalk or interaction between adjacent filters can become significant. Adjacent components in an imaging device must be sufficiently spaced as interactions between filters can distort GMR spectral characteristics in two ways: (1) energy passing through leaks into an adjacent detector and (2) light from one filter interacts with light from an adjacent filter. Figure 4 presents FDTD simulations detailing crosstalk effects in this design. Nominal filter dimensions are used with 631 and $831 \mathrm{~nm}$ periods for filters 1 and 2, respectively. Reference spectra (black) for filter 1 and filter 2 are simulated independently (i.e., a separate simulation without the effects of a second adjacent filter). Figure 4(a) shows the interaction of two adjacent filters as the lateral spacing between them, $w$, is varied. $w$ is defined as the mirror width, while the spacer lengths $s_{1}$ and $s_{2}$ of filters 1 and 2, respectively, are maintained constant. These spectra are presented for $w$ from 0 to $50 \mathrm{~nm}$, in increments of $10 \mathrm{~nm}$ with a separate power monitor (representing a photodetector) located $300 \mathrm{~nm}$ below each filter. We define this distance between the bottom surface of the filters and the location of the power monitors as $L$. In general, although the peak shape is distorted slightly, especially for $w<20 \mathrm{~nm}$, the GMR of each filter maintains its amplitude and FWHM, indicating that crosstalk in this design is minor even at small
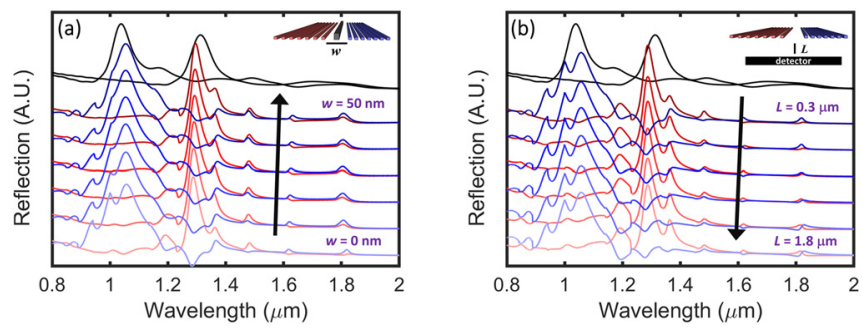

FIG. 4. FDTD-generated spectra demonstrating crosstalk of two adjacent filters as (a) the lateral spacing between them, $w$, and (b) the axial distance between the filter and the monitor, $L$, is varied. Filter 1 (blue) has parameters $a=631 \mathrm{~nm}, h=64 \mathrm{~nm}$, $f=0.18, d=61 \mathrm{~nm}$, and $s=215 \mathrm{~nm}$ and filter 2 (red) has parameters $a=831 \mathrm{~nm}$, $h=64 \mathrm{~nm}, f=0.18, d=61 \mathrm{~nm}$, and $s=215 \mathrm{~nm}$. (a) Overlaid spectra of two filters as $w$ is varied from 0 to $50 \mathrm{~nm}$ in increments of $10 \mathrm{~nm}$, while $L=300 \mathrm{~nm}$. (b) Overlaid spectra of two filters as $L$ is varied from 300 to $1800 \mathrm{~nm}$ in increments of $300 \mathrm{~nm}$, with $w=0$ (i.e., no mirror between filters). In both figures, the black spectra indicate the filters at infinity, where each filter is run in a separate simulation independent of the other filter. values of $w$. Figure 4(b) shows the interaction of two adjacent filters as $L$ is varied from 300 to $1800 \mathrm{~nm}$ in increments of $300 \mathrm{~nm}$, with $w=0 \mathrm{~nm}$ (i.e., no mirror). Minimal change is observed even as the photodetectors/monitors are moved away from the bottom of the filter, indicating that scattering is insignificant in this design. Therefore, GMR peak distortions that appear due to filter proximity result from the coupling of the leaky waveguide mode into the adjacent filter, rather than scattering. This implies the existence of a design with comparable or improved performance without metal mirrors where adjacent filters can freely couple into their neighbors. In this alternatively proposed mirrorless design, a lack of metal mirrors leads to reduced background noise. Thus, we have shown that crosstalk is minimal in this design and potential exists to capitalize on this effect to our advantage.

In summary, conventional GMR-based filters possess high resolving power at the cost of requiring many periods and thus large lateral footprints/pixel sizes in an imaging system, as incident light scattered into a periodic array must propagate for a sufficient number of periods until it scatters back out. Without this, edge loss effects are observed, decreasing peak amplitudes and increasing bandwidths. We demonstrated the suppression of these edge losses in simulation and experiment with a design that utilizes mirrors at the array edges. These $\mathrm{Al}$ reflective mirrors surround a seven-period compact and an ultrathin a-Si grating, all embedded in a continuous $\mathrm{SiO}_{2}$ matrix. The resulting filter demonstrated narrow-band, passively tunable, reflectivity peaks suitable for spectral imaging applications in the near-IR. We compared the effectiveness of this design to an infinite design and a similar compact design without mirrors. We demonstrated passive tunability of the GMR via geometric parameters to obtain the desired spectral characteristics and verified it experimentally. Miniaturization of the GMR filter enables the incorporation of these filters in imaging applications where spatial resolution would otherwise be limited by conventionally large footprints of GMR-based designs.

See the supplementary material for additional details on 2D nanopillar array for polarization-independence, number of periods required to obtain the desired spectral characteristics, the effect of varying period in finite GMR designs, top down schematic of filter array and surrounding reflective frame, ellipsometry, Fresnel correction, and methods.

The authors gratefully acknowledge the financial support of the Department of Defense through J.R.G.'s Vannevar-Bush Faculty Fellowship (No. N00014-16-1-2827) and the critical support and infrastructure provided by the Kavli Nanoscience Institute at Caltech. The authors also thank Harry Atwater for computational resources used to calculate a portion of these results.

\section{DATA AVAILABILITY}

The data that support the findings of this study are available from the corresponding author upon reasonable request.

\section{REFERENCES}

${ }^{1}$ M. Fingas and C. E. Brown, “Oil spill remote sensing: A review," in Oil Spill Science and Technology (Elsevier, 2011), pp. 111-169.

${ }^{2}$ M. Fingas and C. E. Brown, Sensors 18, 91 (2017).

${ }^{3}$ I. Leifer, W. J. Lehr, D. Simecek-beatty, E. Bradley, R. Clark, P. Dennison, Y. Hu, S. Matheson, C. E. Jones, B. Holt, M. Reif, D. A. Roberts, J. Svejkovsky, G. Swayze, and J. Wozencraft, Remote Sens. Environ. 124, 185 (2012). 
${ }^{4}$ D. J. Williams, B. L. Feldman, T. J. Williams, D. Pilant, P. G. Lucey, and L. D. Worthy, Proc. SPIE 5655, 134 (2005).

${ }^{5}$ T. Slonecker, G. B. Fisher, D. P. Aiello, and B. Haack, Remote Sens. 2, 2474 (2010).

${ }^{6}$ Y. Feng and D. Sun, Crit. Rev. Food Sci. Nutr. 52, 1039 (2012).

${ }^{7}$ R. Lu and Y.-R. Chen, Proc. SPIE 3544, 121 (1999).

${ }^{8}$ R. M. Levenson and J. R. Mansfield, Cytometry 69A, 748 (2006).

${ }^{9}$ T. Zimmermann, J. Rietdorf, and R. Pepperkok, FEBS Lett. 546, 87 (2003).

${ }^{10}$ P. W. T. Yuen and M. Richardson, Imaging Sci. J. 58, 241 (2010).

"G. A. Shaw and H. K. Burke, Lincoln Lab. J. 14, 3 (2003), https://courses.cs.washington.edu/courses/cse591n/07sp/papers/Shaw2003.pdf.

${ }^{12}$ N. Gat, S. Subramanian, J. Barhen, and N. Toomarian, Proc. SPIE 2962, 63 (1997).

${ }^{13}$ F. Flory and A. Piegari, Optical Thin Films and Coatings: From Materials to Applications (Woodhead Publishing, Cambridge, 2013).

${ }^{14}$ Y. Huo, C. C. Fesenmaier, and P. B. Catrysse, Opt. Express 18, 5861 (2010).

${ }^{15}$ C. C. Fesenmaier, Y. Huo, and P. B. Catrysse, Proc. SPIE 7250, 72500G (2009).

${ }^{16}$ S. P. Burgos, S. Yokogawa, and H. A. Atwater, ACS Nano 7, 10038 (2013).

${ }^{17}$ S. Yokogawa, S. P. Burgos, and H. A. Atwater, Nano Lett. 12, 4349 (2012).

${ }^{18} \mathrm{Q}$. Chen, D. Chitnis, K. Walls, T. D. Drysdale, S. Collins, and D. R. S. Cumming, IEEE Photonics Technol. Lett. 24, 197 (2012).

${ }^{19}$ D. Inoue, A. Miura, T. Nomura, H. Fujikawa, K. Sato, N. Ikeda, D. Tsuya, Y. Sugimoto, and Y. Koide, Appl. Phys. Lett. 98, 093113 (2011).

${ }^{20}$ Q. Chen and D. R. S. Cumming, Opt. Express 18, 14056 (2010).

${ }^{21}$ B. Zeng, Y. Gao, and F. J. Bartoli, Sci. Rep. 3, 2840 (2013).

${ }^{22}$ T. Xu, Y.-K. Wu, X. Luo, and L. J. Guo, Nat. Commun. 1, 59 (2010).

${ }^{23}$ Y.-T. Yoon and S.-S. Lee, Opt. Express 18, 5344 (2010).

${ }^{24}$ V. R. Shrestha, S. S. Lee, E. S. Kim, and D. Y. Choi, Sci. Rep. 4, 4921 (2014).

${ }^{25}$ K. T. Lee, S. Seo, J. Y. Lee, and L. J. Guo, Appl. Phys. Lett. 104, 231112 (2014).

${ }^{26} \mathrm{~K}$. Hehl, J. Bischoff, U. Mohaupt, M. Palme, B. Schnabel, L. Wenke, R. Bodefeld, W. Theobald, E. Welsch, R. Sauerbrey, and H. Heyer, Appl. Opt. 38, 6257 (1999).

${ }^{27}$ L. Mashev and E. Popov, Opt. Commun. 55, 377 (1985).

${ }^{28} \mathrm{M}$. T. Gale, K. Knop, and R. Morf, SPIE Opt. Secur. Anticounterfeiting Syst. 1210, 83 (1990).

${ }^{29}$ B. H. Cheong, O. N. Prudnikov, E. Cho, H. S. Kim, J. Yu, Y. S. Cho, H. Y. Choi, and S. T. Shin, Appl. Phys. Lett. 94, 213104 (2009).

${ }^{30}$ Y. Kanamori, M. Shimono, and K. Hane, IEEE Photonics Technol. Lett. 18, 2126 (2006).

${ }^{31}$ E.-H. Cho, H.-S. Kim, B.-H. Cheong, O. Prudnikov, W. Xianyua, J.-S. Sohn, D.J. Ma, H.-Y. Choi, N.-C. Park, and Y.-P. Park, Opt. Express 17, 8621 (2009).

${ }^{32}$ D. B. Mazulquim, K. J. Lee, J. W. Yoon, L. V. Muniz, B.-H. V. Borges, L. G. Neto, and R. Magnusson, Opt. Express 22, 30843 (2014).

${ }^{33}$ G. A. Golubenko, A. S. Svakhin, V. A. Sychugov, A. V. Tischenko, E. Popov, and L. Mashev, Opt. Quantum Electron. 18, 123 (1986).
${ }^{34}$ S. S. Wang and R. Magnusson, Appl. Opt. 32, 2606 (1993).

${ }^{35}$ S. S. Wang, R. Magnusson, J. S. Bagby, and M. G. Moharam, J. Opt. Soc. Am. A 7, 1470 (1990).

${ }^{36}$ Y. Ding and R. Magnusson, Opt. Express 15, 680 (2007).

${ }^{37}$ V. Karagodsky, F. G. Sedgwick, and C. J. Chang-Hasnain, Opt. Express 18, 16973 (2010).

${ }^{38}$ C. J. Chang-Hasnain and W. Yang, Adv. Opt. Photonics 4, 379 (2012).

${ }^{39}$ P. Lalanne, J. P. Hugonin, and P. Chavel, J. Light. Technol. 24, 2442 (2006).

${ }^{40}$ P. P. Iyer, N. A. Butakov, and J. A. Schuller, ACS Photonics 2, 1077 (2015).

${ }^{41}$ R. C. Ng, J. C. Garcia, J. R. Greer, and K. T. Fountaine, ACS Photonics 6, 265 (2019).

${ }^{42}$ T. Clausnitzer, J. Limpert, K. Zöllner, H. Zellmer, H.-J. Fuchs, E.-B. Kley, A. Tünnermann, M. Jupé, and D. Ristau, Appl. Opt. 42, 6934 (2003).

${ }^{43}$ D. L. Brundrett, E. N. Glytsis, and T. K. Gaylord, Opt. Lett. 23, 700 (1998).

${ }^{44}$ C. F. R. Mateus, M. C. Y. Huang, L. Chen, C. J. Chang-Hasnain, and Y. Suzuki, IEEE Photonics Technol. Lett. 16, 1676 (2004).

${ }^{45}$ R. Magnusson and Y. H. Ko, Proc. SPIE 9927, 992702 (2016).

${ }^{46}$ R. Magnusson, Opt. Lett. 39, 4337 (2014).

${ }^{47} \mathrm{M}$. Niraula and R. Magnusson, Opt. Lett. 41, 2482 (2016).

${ }^{48}$ M. Niraula, J. W. Yoon, and R. Magnusson, Opt. Lett. 40, 5062 (2015).

${ }^{49}$ J. Liu, M. V. Schulmerich, R. Bhargava, and B. T. Cunningham, Opt. Express 19, $24182(2011)$.

${ }^{50}$ K. Ikeda, K. Takeuchi, K. Takayose, I.-S. Chung, J. Mørk, and H. Kawaguchi, Appl. Opt. 52, 1049 (2013).

${ }^{51}$ D. W. Peters, R. R. Boye, J. R. Wendt, R. A. Kellogg, S. A. Kemme, T. R. Carter, and S. Samora, Opt. Express 35, 3201 (2010).

${ }^{52}$ R. R. Boye and R. K. Kostuk, Appl. Opt. 39, 3649 (2000).

${ }^{53}$ D. K. Jacob, S. C. Dunn, and M. G. Moharam, J. Opt. Soc. Am. A 17, 1241 (2000).

${ }^{54}$ J. M. Bendickson, E. N. Glytsis, T. K. Gaylord, and D. L. Brundrett, J. Opt. Soc, Am. A 18, 1912 (2001).

${ }^{55} \mathrm{~A}$. Liu, W. Hofmann, and D. Bimberg, Opt. Express 22, 11804 (2014).

${ }^{56}$ I.-R. Johansen, J. O. Grepstad, M. M. Greve, O. Solgaard, A. Sudbø, and B. Holst, Opt. Express 21, 23640 (2013).

${ }^{57}$ D. Fleischman, K. T. Fountaine, C. R. Bukowsky, G. Tagliabue, L. A. Sweatlock, and H. A. Atwater, ACS Photonics 6, 332 (2019).

${ }^{58}$ Y. Zhou, M. Moewe, J. Kern, M. C. Huang, and C. J. Chang-Hasnain, Opt. Express 16, 17282 (2008).

${ }^{59}$ S. Ura, S. Murata, Y. Awatsuji, and K. Kintaka, Opt. Express 16, 12207 (2008).

${ }^{60}$ J. H. Barton, R. C. Rumpf, R. W. Smith, C. Kozikowski, and P. Zellner, Prog. Electromagn. Res. B 41, 269 (2012).

${ }^{61}$ C. Audet, S. L. Digabel, C. Tribes, and V. Rochon Montplaisir, "The NOMAD project," software available at https://www.gerad.ca/nomad.

${ }^{62}$ K. Diest, L. A. Sweatlock, and D. E. Marthaler, J. Appl. Phys. 108, 084303 (2010).

${ }^{63}$ S. Le Digabel, ACM Trans. Math. Software 37, 1 (2011). 